\title{
Automatic Generation of RIAs Using RUX-Tool and Webratio*
}

\author{
Marino Linaje ${ }^{1}$, Juan Carlos Preciado ${ }^{1}$, Rober Morales-Chaparro ${ }^{2}$, \\ Roberto Rodríguez-Echeverría, and Fernando Sánchez-Figueroa ${ }^{1}$ \\ ${ }^{1}$ Quercus SEG, Escuela Politécnica, Universidad de Extremadura \\ Avda. de la Universidad S/N, Cáceres, Spain \\ mlinaje@unex.es \\ ${ }^{2}$ R\&D Department, Homeria Open Solutions, S.L. \\ Vivero de Empresas \\ Avda. de la Universidad S/N, Cáceres, Spain \\ robermorales@homeria.com
}

\begin{abstract}
This work describes RUX-Tool, an MDD-based tool that gives support to the modeling and automatic code generation of User Interfaces for Rich Internet Applications (RIAs) with multidevice and multiplatform capabilities. This tool is mainly thought to be used with other tools based on Web methodologies such as it is the case of WebRatio that automatically generates the content structure and the business logic.
\end{abstract}

Keywords: Rich Internet Applications, User Interfaces, CASE Tool.

\section{Introduction}

The future of the Web is being built using technologies such as Rich Internet Applications (RIAs) [1] which, on the one hand, offer all the advantages of traditional Web applications and, on the other hand, add the flexibility and user-friendliness of desktop solutions. RIAs offer online and offline capabilities, sophisticated user interfaces, augmented storage and processing capabilities at the client side, high levels of user interaction, usability and personalization and they minimize bandwidth usage through the separation of presentation from content at the client side.

Some of the most well-known technologies used for developing RIAs are AJAX [5], Flash [5], Flex [5] and OpenLaszlo [5]. However, while RIA technologies are reaching maturity and a growing number of Web 2.0 applications are being developed based on them, there is a lack of comprehensive models and methodologies for the systematic development of RIAs [5].

Many of the RIA features are related with User Interfaces. With no doubt, they are the most appreciated features by users. The main contribution of this paper is introducing a visual and intuitive tool for the design of RIA User Interfaces on top of existing applications developed with a Web methodology. This tool, called RUX-Tool,

* Developed under Spanish Research Projects: TSI-020501-2008-47 and TIN2008-02985. 
gives support to the automatic code generation for different devices (PC, Mobile, PDA, etc) and different platforms (AJAX, Flex, OpenLaszlo).

RUX-Tool works together with WebRatio, the WebML CASE Tool. Once the application has been developed using WebRatio, one can use RUX-Tool to obtain from the WebRatio model the data structure and business logic information. Then, the process of modeling the User Interface begins, as described below.

\section{RUX-Tool}

RUX-Tool is based on RUX-Method [4] (Figure 1). According to [3] the design of the UI is divided into 4 levels: Concepts and Tasks, Abstract Interface, Concrete Interface and Final Interface. In RUX-Method Concepts and Tasks are provided by the underlying Web model and each level is composed by Interface Components which are specified in the RUX Components Library.

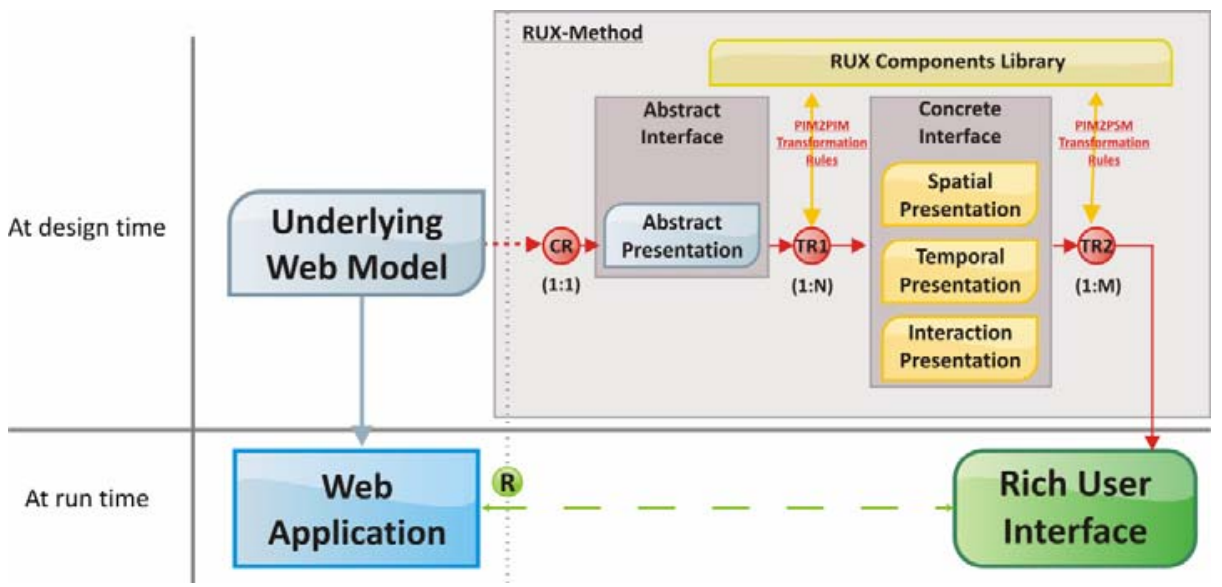

Fig. 1. Design stages in RUX-Method

The development process in RUX-Method has four main stages: connection with the previously defined underlying Web model, definition of the Abstract Interface, definition of the Concrete Interface and specification of the Final Interface which ends in code generation.

So, the first stage in RUX-Tool deals with the connection with the WebRatio model. At this stage, the presentation elements and the relationships among them are extracted, as well as the defined operations on the WebRatio model. On the basis of the results offered by the Connection Rules (CR in Figure 1), RUX-Tool builds the Abstract Interface, which is independent from the platform and the final display device. Applying to the Abstract Interface the first set of Transformation Rules (TR1 in Figure 1), the Concrete Interface, which allows the appearance, spatial arrangement, temporal and interactive behavior, is obtained. Finally, from the Concrete Interface and through the second set of Transformation Rules (TR2 in Figure 1), the Final Interface 


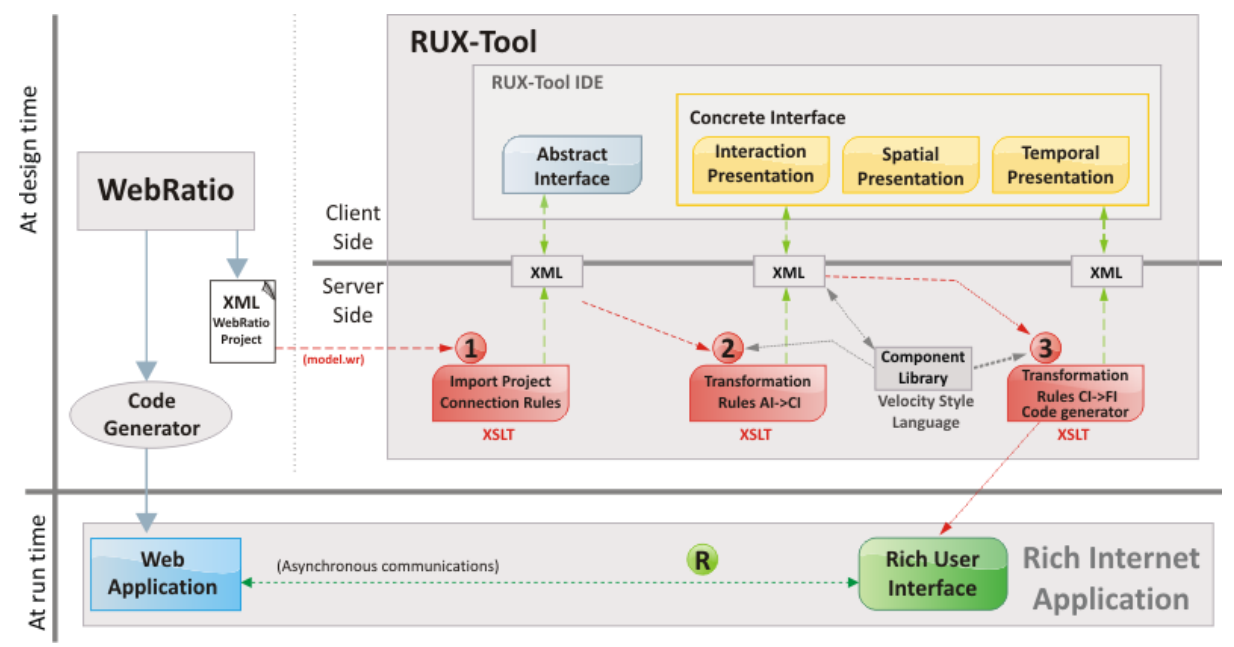

Fig. 2. RUX-Tool architecture

is obtained. The Abstract and the Concrete Interface levels can be modified by the modeler before performing the next stage.

RUX-Tool is not a desktop application. It is itself a RIA, so it is accessed from a browser, and allows working on-line in a cooperative way.

The general architecture of the tool can be seen in Figure 2. RUX-Tool, as a typical RIA, has two sides: client side and server side. The client side includes a graphic UI for redesigning the different levels of User Interfaces once they have been automatically obtained from their corresponding previous stage. The client side also includes different wizards and dialogs to configure the tool, to import the WebRatio project and to specify the chosen devices and platforms for the final code generation.

On the other hand, the server side is in charge of performing the required transformations between interface levels and the connection with the underlying WebRatio application. This implies the communication between client and server sides to interchange the representation of the different interface levels. This is done by means of XML.

The Component Library is also at the server side. It has a plug-in based architecture favoring an easy management of components (deletion, insertion, etc). Each component is implemented using a template based on Velocity Style Language.

\section{RUX-Tool in the Market}

Currently, RUX-Tool is being used with Webratio 5.1. It is being developed between the University of Extremadura and Homeria Open Solutions, (spinoff of the same university) as a consequence of a Spanish Research project from the Industry Department.

RUX-Tool has being used in several projects such as a Virtual Campus, a Content Management System or Virtual Shop Management System. Figure 3 shows a 


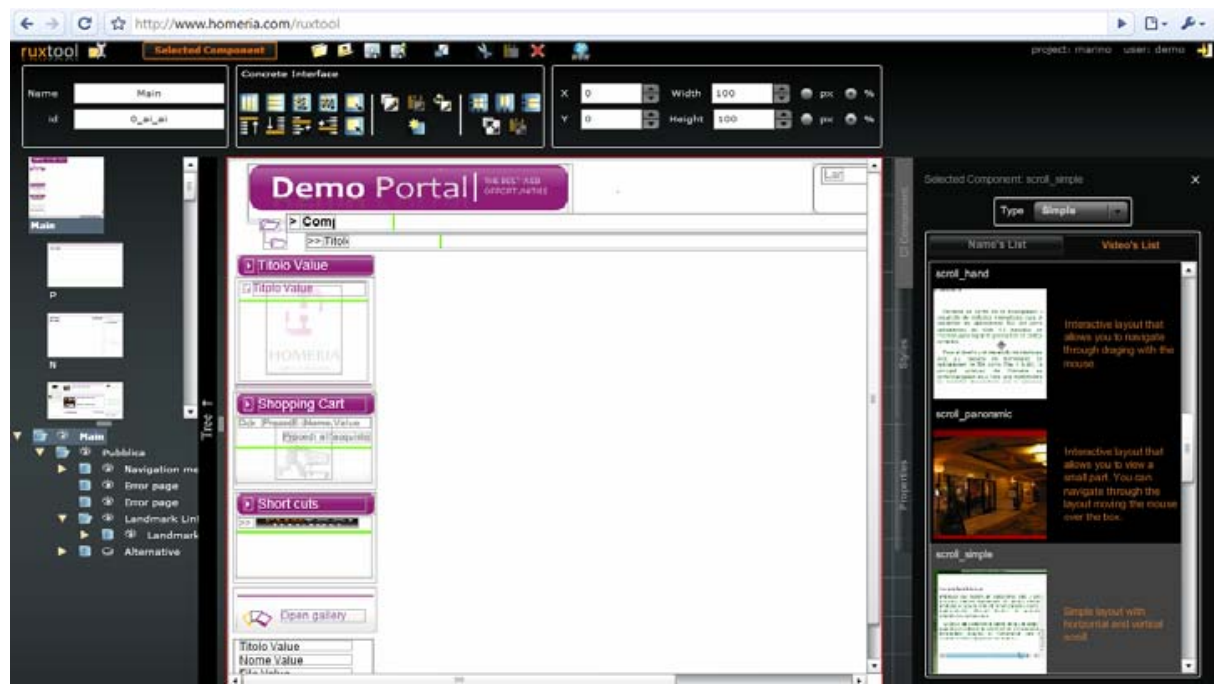

Fig. 3. RUX-Tool

snapshot of the tool at the client side when designing a simple Web portal with a shopping cart.

We refer to the reader to the RUX-project site (www.ruxproject.org) for more details on RUX-Tool (video-demos, examples, etc).

\section{References}

1. Bozzon, A., Comai, S., Fraternali, P., Toffetti Carughi, G.: Conceptual Modeling and Code Generation for Rich Internet Applications. In: International Conference on Web Engineering ICWE, pp. 353-360. ACM, New York (2006)

2. Ceri, S., Fraternali, P., Bongio, A., Brambilla, M., Comai, S., Matera, M.: Designing DataIntensive Web Applications. Morgan Kauffmann, San Francisco (2002)

3. Limbourg, Q., Vanderdonckt, J., Michotte, B., Bouillon, L., Lopez, V.: UsiXML: a Language Supporting Multi-Path Development of User Interfaces. In: Bastide, R., Palanque, P., Roth, J. (eds.) DSV-IS 2004 and EHCI 2004. LNCS, vol. 3425, pp. 207-228. Springer, Heidelberg (2005)

4. Linaje, M., Preciado, J.C., Sanchez-Figueroa, F.: Engineering Rich Internet Application User Interfaces over Legacy Web Models. IEEE Internet Computing 11(6), 53-59 (2007)

5. Preciado, J.C., Linaje, M., Sanchez, F., Comai, S.: Necessity of methodologies to model Rich Internet Applications. In: IEEE Int. Symposium on Web Site Evolution, pp. 7-13 (2005) 